\title{
Associação da carga parasitária renal com achados laboratoriais em cães com leishmaniose visceral
}

\author{
Renal parasite load association with laboratory findings in dogs with visceral leishmaniasis
}

\author{
Mariana de Medeiros Torres ${ }^{\mathrm{I}^{*}}$ Arleana do Bom Parto Ferreira de Almeida \\ Eveline da Cruz Boa Sorte ${ }^{\mathrm{I}}$ Daphine Ariadne Jesus de Paula ${ }^{\mathrm{I}}$ \\ Anderson Castro Soares de Oliveira ${ }^{I I}$ Caroline Argenta Pescador ${ }^{\mathrm{I}}$ \\ Adriane Jorge Mendonça ${ }^{I}$ Luciano Nakazato ${ }^{\mathrm{I}}$
}

- NOTA -

RESUMO

\begin{abstract}
A leishmaniose visceral canina é uma doença grave e a morte ocorre por falência renal, considerando que os métodos diagnósticos convencionais não possibilitam a classificação clínica do animal. $O$ objetivo deste estudo foi associar a carga parasitária renal aos achados clínicos e histopatológicos em cães com leishmaniose visceral. A análise microscópica revelou predomínio de nefrite intersticial mononuclear de graus variados em 59,3\% dos cães avaliados. Entretanto, não houve diferença entre a carga parasitária renal de sintomáticos $e$ oligossintomáticos $(P=0,35)$. As lesões renais foram de ordem inflamatória e a quantidade de parasitos não influenciaram na característica dessas lesões e nem nas alterações bioquímicas, mesmo em cães com diferentes classificações clínicas.
\end{abstract}

Palavras-chave: rim, $q P C R$, cão, Leishmania sp..

\section{ABSTRACT}

Canine visceral leishmaniasis is a severe disease and the death occurs from renal failure, whereas conventional diagnostic methods do not allow the animal clinical staging. The aim of this study was to associate the renal parasite load to clinical and histopathological findings in dogs with visceral leishmaniasis. Microscopic analysis revealed a predominance of mononuclear interstitial nephritis of varying degrees in 59, 3\% of dogs evaluated. However, no difference was found between the renal parasite load of symptomatics and oligosymptomatics $(P=0,35)$. Renal lesions were inflammatory order and amount of parasites not influenced the characteristics of theses lesions nor biochemical changes, even in dogs with different clinical classifications.

Key words: kidney, $q P C R$, dog, Leishmania sp.
A leishmaniose visceral canina (LVC) é uma doença insidiosa grave e frequentemente resulta na morte por falência renal. O cão atua como principal reservatório da Leishmania chagasi para o homem e, embora muitos testes tenham sido desenvolvidos para prover um eficiente método de controle e diagnóstico, os mesmos não são satisfatórios na determinação do estado clínico do animal (COSTAVAL et al., 2007; SARIDOMICHELAKIS, 2009).

Considerando os testes disponíveis para diagnóstico da leishmaniose e os métodos convencionais de avaliação clínica, como análise hematológica, bioquímica e histopatológica, a técnica molecular baseada na PCR quantitativa (qPCR) tem importante valor na detecção, quantificação e diferenciação das espécies de Leishmania, permitindo a detecção de DNA independente do estágio clínico (COSTA-VAL et al., 2007; MANNA et al., 2009).

Como a doença renal se apresenta como uma manifestação final da leishmaniose visceral no cão, baseado na sensibilidade da qPCR o objetivo do estudo foi associar a carga parasitária dos rins aos achados clínicos e histopatológicos em cães com leishmaniose visceral.

Amostras de soro e fragmentos dos rins foram coletadas de 30 cães soro reagentes para leishmaniose visceral (IFI/ ELISA) determinados através de kit Biomanguinhos (FIOCRUZ). Estes

'Departamento de Clínica Médica Veterinária (CLIMEV), Faculdade de Agronomia, Medicina Veterinária e Zootecnia (FAMEVZ), Universidade Federal de Mato Grosso (UFMT), Cuiabá, MT, Brasil. E-mail: marys_torres@ hotmail.com. *Autor para correspondência. "Departamento de Estatística, Instituto de Ciências Exatas e da Terra (ICET), UFMT, Cuiabá, MT, Brasil. 
cães constituíram o Grupo com Leishmaniose Visceral Canina (GLVC), onde a $L$. (L.) chagasi foi caracterizada por PCR- RFLP (ANDRADE et al., 2006) de medula óssea, linfonodo e sangue.

O GLVC consistiu de 15 machos e 15 fêmeas com idade variando de oito meses à 14 anos, das raças Pinscher (4/ 30), Pastor Belga (1/ 30), Pit Bull (6/ 30), Rottweiler (1/ 30) e os sem definição racial (18/ 30). Os cães desse grupo foram classificados clinicamente como sintomáticos, oligossintomáticos e assintomáticos de acordo com MANCIANTI et al. (1988).

Um grupo controle (GC) constituído por 13 cães (nove machos e quatro fêmeas), com idade variando de dois a seis anos, das raças Poodle (3/ 13), Lhasa Apso (3/ 13), Boxer (1/ 13) e os sem raça definida (6/ 13), foi formado para referência dos parâmetros bioquímicos de ureia, creatinina, cálcio, fósforo e albumina. Estes cães foram considerados sadios após exame clínico, laboratorial e PCR para Leishmania sp., Ehrlichia canis e Anaplasma platys.

As amostras de soro obtidas foram submetidas ao teste pelo método cinético/ colorimétrico em analisador semiautomático (CELM SB-190 ${ }^{\circledR}$ ) utilizando kits comerciais para dosagem de ureia, creatinina, cálcio, fósforo e albumina (FINCO, 1997).

Após eutanásia e necropsia dos cães do GLVC dois fragmentos dos rins foram coletados, um fixado em solução de formalina a $10 \%$, processado rotineiramente e corado com hematoxilina e eosina (HE) e outro fragmento para quantificação parasitária por qPCR.

A análise do qCR foi realizada em triplicata através do Sistema de Detecção da Sequência Step One ${ }^{\mathrm{TM}}$ Real- Time PCR Systems (Applied Biosystems) utilizando como alvo a mesma sequência de DNA empregada na PCR convencional. A curva padrão foi estabelecida utilizando quantidades conhecidas de plasmídeos a partir do DNA de Leishmania sp. extraído de 2,8 x $10^{8}$ moléculas/ $\mu \mathrm{L}$, variando de 10 a $10^{7}$ parasitos em cada reação para obter o limite de detecção, de acordo com QUARESMA et al. (2009).

Os resultados obtidos foram submetidos à análise baseados no teste não paramétrico de MannWhitney, teste Tuckey e correlação com auxílio do programa estatístico GraphPad Prism v. 4.0.

No GLVC predominaram os oligossintomáticos (18/30), seguido dos sintomáticos (12/30) com resultados de IFI variando de 1:80 a 1:640, observando maiores titulações em cães sintomáticos. Os achados clínicos mais frequentes nos cães oligossintomáticos foram descamação furfurácea, linfadenomegalia e perda de peso, enquanto os sintomáticos, além destes já citados, apresentavam também anorexia, esplenomegalia, uveíte e caquexia.

MANNA et al. (2009) demonstraram que cães com sintomatologia mais severa produzem anticorpos específicos que podem ser facilmente detectados, indicando uma relação entre a carga parasitária e a manifestação clínica. Entretanto, quando comparadas as cargas parasitárias dos rins $(\mathrm{P}=0,35)$ entre os sintomáticos e oligossintomáticos, não houve diferença, possivelmente como resultado do tamanho da amostra em cada grupo clínico. Essa diferença também pode ser atribuída ao tecido de escolha, já que os parasitos são mais bem estabelecidos na medula óssea, onde as amastigotas tem tempo suficiente para estimular o sistema imune (QUARESMA et al., 2009).

O DNA de Leishmania sp. foi detectado nos rins de 12 cães, seis sintomáticos e seis oligossintomáticos, até a concentração de $0,1 \mathrm{fg} / \mu \mathrm{L}$ semelhante aos resultados obtidos por QUARESMA et al. (2009), evidenciando a alta sensibilidade desta técnica molecular o que possibilita determinar a extensão de infecções subclínicas, fator importante na detecção da doença principalmente em assintomáticos. Apesar da ampla variação, grandes quantidades de parasitos foram encontradas no tecido renal.

Quando comparados sintomáticos e oligossintomáticos, não houve diferença nos resultados da bioquímica sérica. As alterações encontradas no GLVC foram hipoalbuminemia, hiperfosfatemia com consequente hipocalcemia e aumento nos níveis séricos de ureia e creatinina, resultados já amplamente relatados na leishmaniose visceral canina devido à inflamação crônica e doença renal concomitante (SARIDOMICHELAKIS, 2009; CORTADELLAS et al., 2010).

$\mathrm{Na}$ histopatologia, os rins apresentaram lesões inflamatórias e degenerativas. Dez cães $(33,33 \%)$ não apresentaram alterações renais, nos demais, a presença de proteína (18,51\%), degeneração tubular multifocal leve $(3,70 \%)$, nefrite intersticial mononuclear em graus variados $(59,25 \%)$, glomerulonefrite membranosa $(18,51 \%)$ e fibrose intersticial e periglomerular $(18,51 \%)$ também descritas por COSTA et al. (2003), encaminharam o diagnóstico para insuficiência renal, em associação aos achados bioquímicos. A relação das cargas parasitárias dos animais do GLVC, com a presença de lesões histopatológicas e alterações bioquímicas está exposta na tabela 1 . 
Tabela 1 - Valores médios da carga parasitária renal (CPR) e bioquímica sérica de acordo com a lesão histopatológica encontrada nos rins de cães com leishmaniose visceral (GLVC).

\begin{tabular}{lll}
\hline Variável & Não & \\
\hline & Sim & \\
CPR & $4,9^{\mathrm{a}}$ & $4,1^{\mathrm{a}}$ \\
Albumina & $1,5^{\mathrm{a}}$ & $1,7^{\mathrm{a}}$ \\
Cálcio & $9,3^{\mathrm{a}}$ & $9,4^{\mathrm{a}}$ \\
Creatinina & $1,4^{\mathrm{a}}$ & $1,2^{\mathrm{a}}$ \\
Fósforo & $4,2^{\mathrm{a}}$ & $4,8^{\mathrm{a}}$ \\
Ureia & $96,5^{\mathrm{a}}$ & $52,8^{\mathrm{b}}$ \\
\hline
\end{tabular}

Médias seguidas de mesma letra na linha não diferem entre si ao nível nominal de 5\% de significância pelo teste de MannWhitney.

Não houve relação entre a carga parasitária com os achados de IFI e as diferentes características histopatológicas descritas $(p=0,82)$. COSTA et al. (2003) consideram que a nefropatia está mais associada à deposição de imunocomplexos na membrana basal glomerular e ao envolvimento da resposta imune celular. Entretanto, SOLANO-GALLEGO et al. (2007), encontraram DNA de Leishmania na urina de cães com insuficiência renal, evidenciando a presença do parasito no trato urinário de cães clinicamente doentes.

Este estudo buscou correlacionar a presença de parasitos nos rins ao desenvolvimento de alterações clínicas e laboratoriais. A qPCR no tecido investigado mostrou-se altamente sensível. As lesões renais decorrentes da LVC são de ordem inflamatória e a quantidade de parasitos não influencia na característica dessas lesões e nas alterações bioquímicas, mesmo em cães com diferentes classificações clínicas, de forma que a inflamação e a vasculite decorrentes da infecção podem influenciar mais no surgimento de tais alterações do que a presença do parasito no órgão. No entanto, como a insuficiência renal é a principal causa de morte na leishmaniose, é importante investigar os fatores que contribuem para o desenvolvimento da doença renal nestes cães.

\section{COMITÊ DE ÉTICA}

O protocolo do estudo foi aprovado pelo Comitê de Ética em Pesquisa Animal (CEPA: 23108.014734/10-0) da Universidade Federal de Mato Grosso.

\section{REFERÊNCIAS}

ANDRADE, H.M et al. Use of PCR-RFLP to identify Leishmania species in naturally-infected dogs. Veterinary Parasitology, n.
140, p.231-238, 2006. Disponível em: <http://www.sciencedirect. com/science/article/pii/S0304401706002299>. Acesso em: 30 mar 2012. doi: 10.1016/j.vetpar.2006.03.031.

CORTADELLAS, O et al.. Calcium and phosphorus homeostasis in dogs with spontaneous chronic kidney disease at different stages of severity. Journal of Veterinary Internal Medicine, n. 24, p.73-79, 2010. Disponível em:<http://onlinelibrary.wiley.com/ doi/10.1111/j.1939-1676.2009.0415.x/article>. Acesso em: $30 \mathrm{mar}$ 2012. doi: 10.1111/j.1939-1676.2009.0415.x.

COSTA, F.A. et al. Histopathologic patterns of nephropathy in naturally acquired canine visceral leishmaniasis. Veterinary Pathology, n. 40, p.677- 684, 2003. Disponível em: <http://vet. sagepub.com/content/40/6/677.full.pdf+html>. Acesso em: 27 mar 2012. doi: 10.1354/vp.40-6-67.

COSTA-VAL, A.P. et al., Canine visceral leishmaniasis: Relationships between clinical status, humoral immune response, haematology and Lutzomyia (Lutzomyia) longipalpis infectivity. Veterinary Journal, n. 174, p. 636-643, 2007. Disponível em: <http://www.sciencedirect.com/science/article/pii/ S1090023306002450>. Acesso em: 29 mar 2012. doi: 10.1016/j. tvj1.2006.11.006.

FINCO, D.R. In:__ KANEKO, J.J., HARVEY, J.W., BRUSS, M.L. Clinical Biochemistry of Domestic Animals. 5 ed. California: Academic Press, 1997 p. 441-80.

MANCIANTI, F. et al. Studies on canine leishmaniasis control. 1. Evolution of infection of different clinical forms of canine leishmaniasis following antimonial treatment. Transactions of the Royal Society of Tropical Medicine and Hygiene, n. 82, p. 566-567, 1988. Disponível em: <http://www.sciencedirect.com/ science/article/pii/003592038890510X>. Acesso em: 28 mar 2012. doi: 10.1016/0035-9203(88)90510-x.

MANNA, L. et al. Evidence for a relationship between Leishmania load and clinical manifestations. Research in Veterinary Science, n.1, p. 76-78, 2009. Disponível em: <http://www.ncbi.nlm.nih. gov/pubmed/19178919>. Acesso em: 28 mar 2012. doi:10.1016/j. rvsc.2008.12.009.

QUARESMA, P.F. et al., Molecular diagnosis of canine visceral leishmaniasis: Identification of Leishmania species by PCRRFLP and quantification of parasite DNA by real-time PCR. Acta Tropica, n.111, p. 289-294, 2009. Disponível em: <http://www. sciencedirect.com/science/article/pii/S0001706X09001193〉. Acesso em 30 mar 2012. doi: 10.1016/j.actatropica.2009.05.008.

SARIDOMICHELAKIS, M.N. Advances in the pathogenesis of canine leishmaniosis: epidemiologic and diagnostic implications. Veterinary Dermatology, n. 20, p. 471-489, 2009. Disponível em: <http://onlinelibrary.wiley.com>. Acesso em: 30 mar 2012. doi: 10.1111/j.1365-3164.2009.00823.x.

SOLANO-GALLEGO, L et al. Detection of Leishmania infantum DNA by fret-based real-time PCR in urine from dogs with natural clinical leishmaniosis. Veterinary Parasitology, n.147, p. 315319, 2007. Disponível em: <http://www.sciencedirect.com/ science/article/pii/S0304401707002336>. Acesso em 30 mar 2012. doi: 10.1016/j.vetpar.2007.04.013. 